Research Article

\title{
Chest Computerized Tomography Images under Iterative Model Reconstruction Algorithm in Patients with Lung Cancer
}

\author{
Jie Li $\mathbb{D},{ }^{1}$ Wei Wang $\mathbb{D},{ }^{2}$ Shizhi Long $\mathbb{D},{ }^{2}$ Xin Liu $\mathbb{D},{ }^{1}$ Long Huang $\mathbb{D},{ }^{1}$ and Yuanxun Li ${ }^{1}$ \\ ${ }^{1}$ Department of Cardiothoracic Surgery, The Fifth Affiliated Hospital (Zhuhai) of Zunyi Medical University, Zhuhai 519100, \\ Guangdong, China \\ ${ }^{2}$ Department of Imaging, The Fifth Affiliated Hospital (Zhuhai) of Zunyi Medical University, Zhuhai 519100, Guangdong, China
}

Correspondence should be addressed to Jie Li; 2021zyxwk@zmu.edu.cn

Received 25 July 2021; Revised 12 September 2021; Accepted 15 September 2021; Published 7 October 2021

Academic Editor: Gustavo Ramirez

Copyright (C) 2021 Jie Li et al. This is an open access article distributed under the Creative Commons Attribution License, which permits unrestricted use, distribution, and reproduction in any medium, provided the original work is properly cited.

\begin{abstract}
To explore the effect of the full iterative model reconstruction algorithm (IMR) on chest CT image processing and its adoption value in the clinical diagnosis of lung cancer patients, multislice spiral CT (MSCT) scans were performed on 96 patients with pulmonary nodules. Reconstruction was performed by hybrid iterative reconstruction (iDose4) and IMR2 algorithms. Then, the image contrast, spatial resolution, density resolution, image uniformity, and noise of the CT reconstructed image were recorded. The benign and malignant pulmonary nodules of patients were collected and classified into malignant pulmonary nodule group and benign pulmonary nodule group, and the differences in chest CT imaging characteristics between the two groups were compared. The subject's receiver operating characteristic (ROC) curve was used to analyze the diagnostic sensitivity, specificity, and area under the curve (AUC) of CT for benign and malignant pulmonary nodules. It was found that the spatial resolution, density resolution, image uniformity, and contrast of the CT image reconstructed by the IMR2 algorithm were remarkably greater than those of the iDose4 algorithm, and the noise was considerably less than that of the iDose4 algorithm $(P<0.05)$. Among 96 patients with pulmonary nodules, 65 were malignant nodules, including 15 squamous cell carcinoma, 31 adenocarcinoma, and 19 small cell carcinomas. There were 31 cases of benign nodules, including 14 cases of hamartoma, 10 cases of tuberculous granuloma, 2 cases of sclerosing hemangioma, and 5 cases of diffuse lymphocyte proliferation. The pulmonary nodule malignant group and the pulmonary nodule benign group had statistical differences in pulmonary nodule size, nodule morphology, burr sign, lobular sign, vascular sign, bronchial sign, and pleural depression sign $(P<0.05)$. The sensitivity, specificity, and area under the curve (AUC) of IMR2 algorithm processing chest CT images for liver cancer diagnosis were 85.7\%, 82.3\%, and 0.815, respectively, which were significantly higher than the original CT images $(P<0.05)$. In short, chest MSCT based on the IMR2 algorithm can greatly improve the diagnosis efficiency of lung cancer and had practical significance for the timely detection of early lung cancer.
\end{abstract}

\section{Introduction}

Lung cancer is a kind of malignant tumor that occurs in the lungs. It is one of the most common malignant tumors in the world, and its incidence ranks first in the cause of death among tumor diseases in China. Lung cancer is classified into non-small cell lung cancer (NSCLC) and small cell lung cancer (SCLC) according to the pathological type. Among them, NSCLC includes adenocarcinoma (AC), squamous cell carcinoma (SCC), and large cell carcinoma (LCC). NSCLC accounts for more than $85 \%$ of all lung cancers $[1,2]$.
At present, lung cancer is considered as a respiratory disease, and about $80 \%$ of lung cancers are mainly caused by smoking. Global air pollution is also one of the main causes of lung cancer-related deaths [3]. The key to reducing lung cancer mortality lies in early detection. However, early lung cancer often has no obvious clinical symptoms, and health examinations are needed to achieve early diagnosis. Therefore, it is necessary to diagnose and treat lung cancer as early as possible.

As one of the main methods for early diagnosis of lung cancer, chest computerized tomography (CT) has the 
advantages of high spatial resolution, high density resolution, and good anisotropy, but there are also a series of problems that need to be optimized, such as noise and artifacts [4]. In recent years, many advances have been made in the optimization technology for CT images. The reconstruction algorithm for CT images was originally developed by the filtered back projection (FBP) algorithm. FBP algorithm uses filtering function or convolution algorithm to balance the spatial resolution and noise of the final reconstructed image. Due to the high requirements for the amount of projection data, this algorithm makes the CT scan dose reduced, the reconstruction time is too long, and the CT image has problems such as noise [5]. To break through the limitations of the FBP algorithm, people began to try new optimization algorithms, such as adaptive statistical iterative reconstruction (ASiR) [6], iterative reconstruction in image space (IRIS) [7], adaptive iterative dose reduction (AIDR) [8], and hybrid iterative reconstruction technology (iDose).

As an iterative reconstruction algorithm using dual models, iDose4 is part of the iterative technology based on FBP. This algorithm can significantly reduce the noise of CT images, but it is still a part of iterative technology. When image reconstruction is performed, the influence of the system hardware and the photon characteristics of part of the incident X-ray on the image are not considered, which limits the further optimization of CT images [9]. The knowledge based IMR is a new upgrade based on iDose4, which removes the FEP component. The optimization effect is realized through continuously optimizing image statistics, data statistics, and system models in the image and data space. In recent years, a comparative study on the IMR algorithm and the FEP algorithm pointed out that, compared to FEP and IMR, it can reduce the radiation dose by $60 \%$ to $80 \%$, reduces noise by $70 \%$ to $90 \%$, and increases the spatial resolution by about $50 \%$ [10]. It is verified that the IMR algorithm has a good application prospect in the optimization of CT images. At present, the IMR algorithm has been applied to the clinical diagnosis of many diseases, but there is no report about the adoption value of IMR in the early diagnosis of lung cancer patients.

Therefore, the IMR algorithm based on the iDose4 algorithm was employed to iteratively reconstruct the chest $\mathrm{CT}$ of lung cancer patients. The receiver operating characteristic (ROC) curve was used to analyze the early diagnosis value of the two algorithms for lung cancer patients, so as to comprehensively evaluate the application value of the IMR algorithm in the CT diagnosis of lung cancer patients. It was hoped to provide some reference for the related research on the optimization of chest CT image quality and the improvement of diagnostic accuracy in clinical lung cancer patients.

\section{Materials and Methods}

2.1. Research Object. In this study, 96 patients with lung cancer admitted to hospital were selected as the research object, including 54 males and 42 females, aged 36-72 years. All patients received multislice spiral CT (MSCT) scans. The average age of patients was $58.05 \pm 10.95$ years, the body mass index (BMI) ranged from $19.70-29.64 \mathrm{~kg} / \mathrm{m}^{2}$, and the average BMI was $26.35 \pm 3.67 \mathrm{~kg} / \mathrm{m}^{2}$. The benign and malignant pulmonary nodules of patients were collected and classified into malignant pulmonary nodule group and benign pulmonary nodule group. The study had been approved by the ethics committee of the hospital. The patients included in the study and their family members were informed and signed the informed consent forms.

Inclusion criteria: (i) the chest CT showed a lung mass with a diameter less than or equal to $3 \mathrm{~cm}$, with clear or fuzzy edges, and a circular-like density with increased density; (ii) complete basic clinical information; (iii) patients without serious heart, liver, or kidney dysfunction and serious infection; (iv) patients without previous history of tumors or other types of tumors. Exclusion criteria: (i) patients who had received chemotherapy and radiotherapy, immunotherapy, and other antitumor treatments before surgery; (ii) patients with other malignant tumors; (iii) female patients during menstruation, pregnancy, and lactation.

2.2. CT Scans. MSCT scans were performed on 96 patients with pulmonary nodules. The CT model was a microplate 3D iCT (Philips Corporation, Cleveland, USA). Breath-hold training was made before plain CT scan of the chest. During the scan, the subject was in a supine position with his arms raised and his head advanced. Scanning conditions: $120 \mathrm{Kv}$, $30 \mathrm{mAs}$ for malignant pulmonary nodule group; $120 \mathrm{Kv}$, $10 \mathrm{mAs}$ for benign pulmonary nodule group. Collimation was $128 \times 0.625 \mathrm{~mm}$, pitch was 0.992 , rotation time was $0.75 \mathrm{~s} / \mathrm{r}$, and matrix was $512 \times 512$. The scan range was from the tip of the lung to the bottom of the lung. Scanning parameters: pitch was 0.920 , stereoscopic field of view (SFOV) was $50 \mathrm{~cm}$, rotation time was $1 \mathrm{~s} / \mathrm{r}$, and collimation was $64 \times 0.625 \mathrm{~mm}$. The tube voltage was $120 \mathrm{kV}$, the tube current was set in 9 groups, and the current sizes were 20, 40, $60,80,100,200,300,400$, and $500 \mathrm{mAs}$, respectively.

\subsection{Patient CT Optimization Based on iDose4 Algorithm and} IMR Algorithm. The original data of the CT images obtained from the scan were reconstructed by the iDose4 algorithm and the IMR algorithm, respectively. The data parameters of iDose4 were Standard (B). iDose was employed to iteratively reconstruct the $4^{\text {th }}$ level among the 7 levels, which was recorded as iDose4. IMR iteratively reconstructed the second level of the 3 levels, which was denoted as IMR 2. The reconstruction parameters were Routine, Soft, and SharpPlus. Display field of view (DFOV) was $25 \mathrm{~cm}$, layer thickness was $5 \mathrm{~mm}$, layer spacing was $5 \mathrm{~mm}$, and matrix was $512 \times 512$. The reconstructed image data was saved in a disc. Image detection was performed by Iris image quality automatic detection software (V2.4, 2009, Iris QA, LLC, USA).

The IMR algorithm model is upgraded and optimized based on the image degradation model. First, $p m \times n L R$ observation images are set.

$$
y_{k}=D B_{k} z+n_{k}, 1 \leq k \leq p .
$$


In the model of (1), $y_{k}$ is an $N \times 1(N=m n)$ vector composed of $k m \times n$ LLR observation images sorted by dictionary. If $r_{1}$ and $r_{2}$ are downsampling factors in the horizontal and vertical directions, respectively, then $Z$ is the $z-a$ vector of the $\mathrm{HH}$ image formed after dictionary sorting, and $r_{1} m \times r_{2} n$ is the affine transformation matrix. $B k$ is the fuzzy matrix, $D$ is the downsampling matrix of $r_{1} r_{2} N \times r_{1} r_{2} N$, and $n_{k}$ is the Gaussian white noise of $N \times 1$ vector, which can be obtained from model (1). The image SSR reconstruction needs to solve the following minimization problems, and the corresponding equation is as follows:

$$
\min J(z): J(z)=\sum_{k}\left\|y_{k}-D B_{k} M_{k} z\right\|^{2} .
$$

Another balance item is introduced for adjustment, and there is

$$
\min J(z): J(z)=\sum_{k}\left\|y_{k}-D B_{k} M_{k} z\right\|^{2}+\lambda_{I}(z) .
$$

In (3), $\lambda_{1}$ is the balance factor and $\rho(z)$ is the regularization term. There are many ways to determine $\rho(z)$.

$$
\rho_{\mathrm{BTV}}(z)=\sum_{l=-w}^{w} \sum_{m=0}^{w} a^{|m|+l l}\left\|z-S_{x}^{l} S_{y}^{m} z\right\|^{2},
$$

where $S_{x}^{l}$ and $S_{y}^{m}$ are the movement matrixes that move the image $z$ in the horizontal and vertical directions by $l$ and $m$ pixels, respectively, and $\alpha(0<\alpha<1)$ is the weighting coefficient.

In short, a constant $\alpha$ will have a double effect on the reconstructed HR image, so how to choose a suitable $\alpha$ is very important for the quality of the final reconstructed $\mathrm{HR}$ image. An adaptive weighting coefficient $\alpha i$ is introduced. According to the difference between the specific edge and the smooth part, the edge of the image is sharpened, and the other parts of the image are also smoothed and noise is suppressed, so as to effectively ensure the image quality. The specific method is as follows.

It is assumed that (5) can be obtained from (4):

$$
\rho_{\mathrm{BTV}}(z)=\sum_{l=-w}^{w} \sum_{m=0}^{w} a^{|m|+|l|}\|z-z l(l, m)\|^{2},
$$

$z-z^{\prime}(l, m)=\left[z_{1}(l, m), z_{2}(l, m), \ldots, z_{r_{1} m \times r_{2} n}(l, m)\right]$ shall have a physical meaning. The element $z_{i}(l, m)$ in the vector is the difference between the pixel $z$ at the same position in the reconstructed image $z-z^{\prime}(l, m)$ and the surrounding pixels. In a grayscale image, if it is too large, it means that the reconstructed image $z$ has a sudden change around the $i$-th pixel; that is, there is an edge around the $i$ point. Therefore, it is hoped that the reconstruction algorithm can sharpen the edge around the $i$ point and highlight the display effect of the edge. Otherwise, it is too small, indicating that the $i$ pixel in the image is very smooth, and it is hoped that the reconstruction algorithm can suppress noise. In summary, to perform SSR reconstruction according to the characteristics of different images, an adaptive bilateral full-variable regularization term is introduced, which is expressed as follows. $z-z \prime(l, m)=\left[z_{1}(l, m), z_{2}(l, m), \ldots, z_{r_{1} m \times r_{2} n}(l, m)\right]$ shall have a physical meaning. The element $z-z_{l}(l, m)$ in the vector $z_{i}(l, m)$ is the difference between the pixel at the same position in the reconstructed image $z$ and the pixels around it. In a grayscale image, $0 \leq z_{i}(l . m) \leq 255$, if $z_{i}$ is too large, it indicates that the reconstructed image $z$ has a sudden change around the first pixel; that is, there is an edge around the $-i$ point. Therefore, it is hoped that the reconstruction algorithm can sharpen the edge around the ip point to highlight the display effect of the edge. Otherwise, $z_{i}(l . m)$ is too small, which means that the $i$ pixel of the image is very smooth, and it is hoped that the reconstruction algorithm can suppress noise. In summary, to realize SSR reconstruction based on the characteristics of different images, an adaptive bilateral full variation regularization term $\rho_{\mathrm{SBTV}}(z)$ is introduced as follows.

$$
\rho_{\mathrm{BTV}}(z)=\sum_{l=-w}^{w} \sum_{m=0}^{w}\left\{\sum_{i=1}^{r_{1} m \times r_{2} n}\left(a_{i}^{|m|+|l|} z_{i}(l, m)\right)^{2}\right.
$$

Equation (6) is the adaptive weighting coefficient that changes according to the difference of each pixel of the reconstructed HR image, which fully guarantees the adaptability of the algorithm, thereby theoretically improving the quality of the reconstructed HR image.

The computer automatically analyzed and detected the CT scan data of 96 patients, and the computer gave a test report. Measurement indicators included spatial resolution, density resolution, image uniformity, and noise conditions.

2.3.1. Spatial Resolution Measurement. It was known that the CT of the patient contained 21-line pairs for visual inspection, which were used as the point source for the software to calculate the modulation transfer function (MTF). First, some images were randomly selected during the preliminary experiment, and the difference between the visual inspection method and the MTF method in evaluating the spatial resolution was compared. Giving that the corresponding value of MTF10\% was close to the visual method, it was set as the reference value of image spatial resolution. In this study, if the tube current was less than $50 \mathrm{mAs}$, since the software cannot automatically detect the point source, it would be sent to the postprocessing workstation. The data cannot be collected accurately, so visual inspection was used to record the line logarithm. This operation was jointly completed by two radiologists with more than 5 years of work experience, and the differences in the judgment results were negotiated and handled.

2.3.2. Density Resolution Measurement. In the Iris image quality automatic detection report, the density resolution is expressed by contrast, which is the minimum CT value required to distinguish a target of a certain size. In this experiment, the contrast of the $\mathrm{CT}$ value required to distinguish a target with a diameter of $5 \mathrm{~mm}$ was included as an objective indicator of the density resolution (unit: \%). 
2.3.3. Image Uniformity and Noise Measurement. In the chest CT image, the software automatically identified and delineated the region of interest and measured the CT value and noise (SD) of the center, upper, and lower left and $2 \mathrm{~cm}$ right away from the image edge. The maximum deviation between edge and center CT value was the reference value of image uniformity. The ambient and central noise values were averaged as the noise of this image.

2.4. Acquisition of CT Imaging Omics Features and Detection of Optimized Effects in Patients with Lung Cancer. Imaging analysis was performed on the optimized CT images of lung cancer patients. The statistics on the typical imaging characteristics of lung cancer patients were collected, and the malignant group of pulmonary nodules and the benign group of pulmonary nodules were analyzed in terms of the imaging characteristics of the CT images of the two groups of patients. Moreover, the differences in the imaging characteristics of the CT images of the two groups of patients were compared, including (i) nodule size: the maximum axial diameter of the nodule in the mediastinal window was measured; (ii) nodule morphology: regular or irregular; (iii) fuzzy sign: it was manifested as a long line shadow or short dense thin line shadow that extended radially from the edge of the nodule to the surrounding lung field in the lung window and was not connected to the pleura; (iv) lobular sign: manifested as obvious unevenness on the edge of the nodule, and multiple arcs with notches; (v) vascular sign: it was manifested as the vascular structure around the nodule gathering in the direction of the lesion, or it was manifested as the blood vessel passing through or ending at the lesion; (vi) bronchial sign: mainly manifested as thin strips, tubular shadows with gas-like density, or dotlike translucent shadows appearing on continuous layers; (vii) cavitation sign: mostly $2-3 \mathrm{~mm}$ gas sample density shadows, excluding the density reduction shadows that appeared on continuous layers, which may be unoccluded small bronchi or alveoli that became larger lumens or vacuoles due to traction; (viii) pleural depression sign: manifested as a linear depression or triangular depression between the tumor lesion and the pleura.

Then, the ROC curve was used to analyze the differences in the sensitivity, specificity, and AUC area of the two algorithms in the diagnosis of benign and malignant pulmonary nodules, so as to measure the optimization value of the algorithm.

Then, the sensitivity, specificity, and AUC of the two algorithms in the diagnosis of benign and malignant pulmonary nodules were analyzed by ROC curve, so as to measure the optimization value of the algorithm. The sensitivity and specificity are calculated by the following equations, respectively:

$$
\begin{aligned}
& \text { Sensitivity }=\frac{|A \cap C|}{|A|}, \\
& \text { Specificity }=\frac{|B \cap D|}{|B|} .
\end{aligned}
$$

A is the degree of coincidence between fitting the real lung cancer lesion area (set as $A$ ) and the lung cancer lesion area determined by the full model iterative algorithm (set as $C) . B$ and $D$ represent other positions outside the real cancer lesion area of lung cancer and other positions outside the cervical cancer lesion area determined by artificial intelligence algorithm, respectively. In addition, three overlapping tumor regions were divided into different structural groups of lung cancer tumors that were, whole-area tumors (WT), core tumor areas (CT), and enhanced tumor areas (ET).

2.5. Statistical Methods. SPSS 19.0 was employed for data statistics and analysis. Percentage (\%) was how count data were expressed, and mean plus or minus standard deviation $(\bar{x}+s)$ was how measurement data were expressed. The comparison of the means between the groups was performed by $t$-test. The count data was expressed as a percentage (\%), and the $\chi^{2}$ test was used. The Mann-Whitney $U$ test was used to compare the differences in the macroscopic characteristics and imaging omics characteristics of the two types of lung cancer patients, and $P<0.05$ indicated that the differences were statistically significant.

\section{Results}

3.1. Comparison of CT Images Processed by Different Algorithms. Figure 1 was a chest CT image of a 67-year-old patient. The noise of CT images of lung cancer patients after iDose 4 algorithm processing was improved in contrast to the CT images of patients before processing, and the image clarity was also improved to a certain extent. The CT image processed by the IMR 2 algorithm was significantly improved compared to the one before the processing. In addition, the IMR algorithm had a better processing effect.

\subsection{Evaluation of CT Image Quality Based on Different} Algorithms. Figures 2-5 show the comparisons of the spatial resolution, density resolution, image uniformity, and noise of the iDose4 algorithm and the IMR algorithm under different parameters, respectively. The spatial resolution, density resolution, image uniformity, and contrast of the CT image reconstructed by the IMR2 algorithm were significantly greater than those of the iDose 4 algorithm, and the noise was notably less than that of the iDose 4 algorithm $(P<0.05)$. Through analysis of variance (ANOVA) test, iDose iterative reconstruction cannot improve the spatial resolution of CT images when the reconstruction parameter was Standard (B). The spatial resolution detection results of IMR and iDose were dramatically different $(P<0.05)$. The spatial resolution of IMR 2 was improved by an average of $16 \%$ in contrast to that of iDose 4 . In IMR iterative reconstruction, the spatial resolution of SharpPlus was greatly higher than that of Routine and Soft, and the spatial resolution of Routine was remarkably superior to that of Soft. The differences among the three groups were considerable through ANOVA test $(P<0.05)$. 


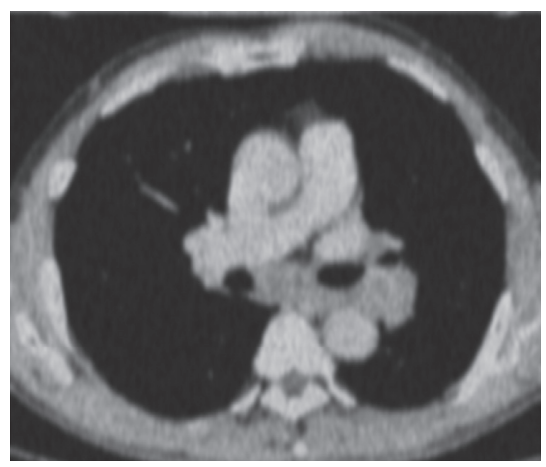

(a)

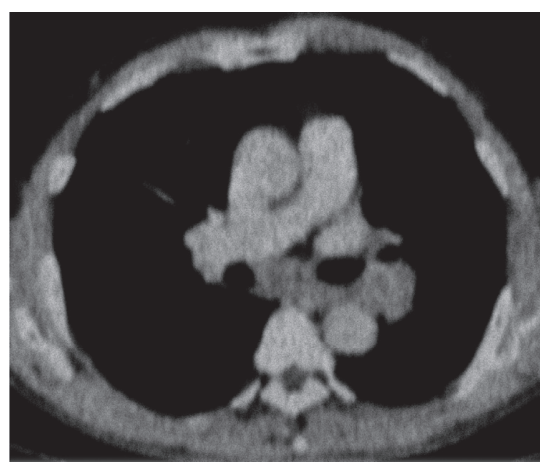

(b)

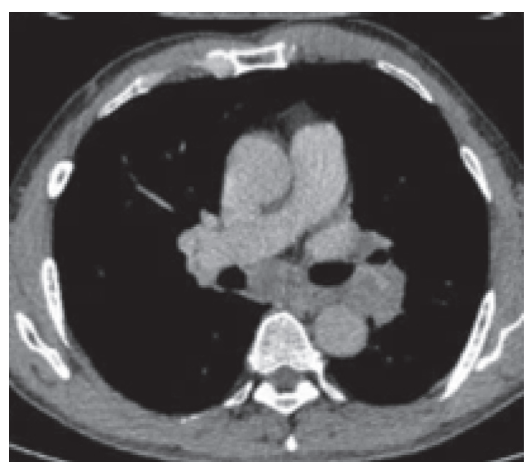

(c)

Figure 1: Comparison of patient's chest CT before and after processing by the two algorithms. (a) Before processing, (b) iDose 4 algorithm processing, and (c) IMR algorithm processing.

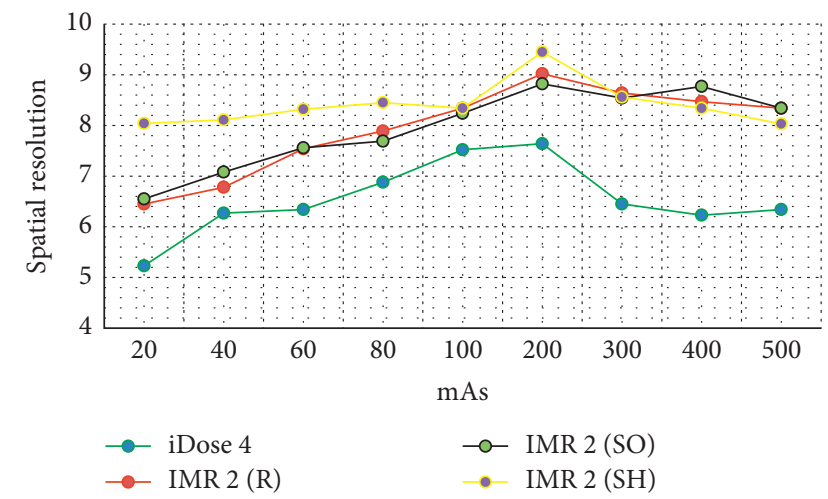

Figure 2: Comparison of image spatial resolution of the two algorithms. (R) denotes Routine, (SO) denotes Soft, and (SH) denotes SharpPlus.

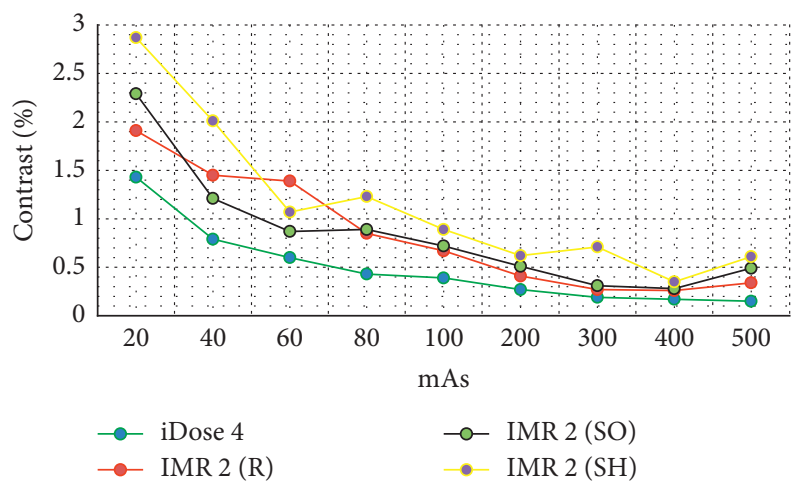

FIgURE 3: The effect of reconstruction of two algorithms on the contrast of CT images. (R) denotes Routine, (SO) denotes Soft, and (SH) denotes SharpPlus.

3.3. Comparison of CT Image Optimization Effects of Two Algorithms. Based on the detected sensitivity, specificity, and AUC, the optimization effect of the patient's CT image after the iDose4 algorithm and IMR2 algorithm reconstruction and optimization processing is shown in Figures 6 and 7. The sensitivity, specificity, and AUC of the patient's

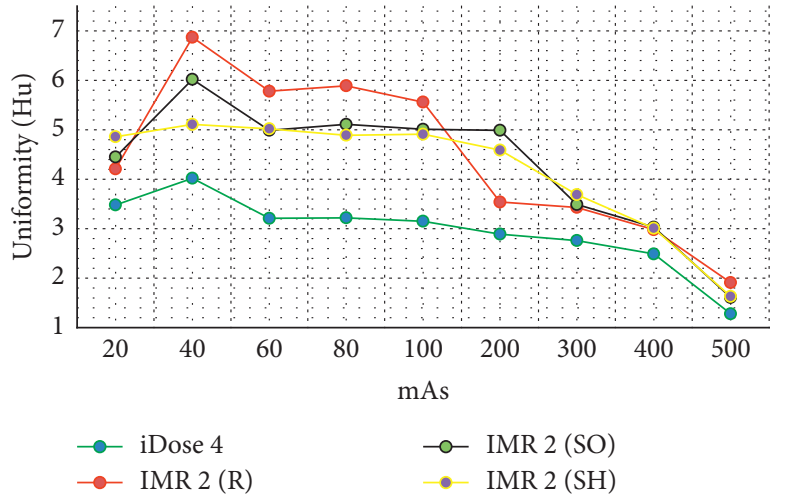

FIgURE 4: The impact of two algorithms on image uniformity. (R) denotes Routine, (SO) denotes Soft, and (SH) denotes SharpPlus.

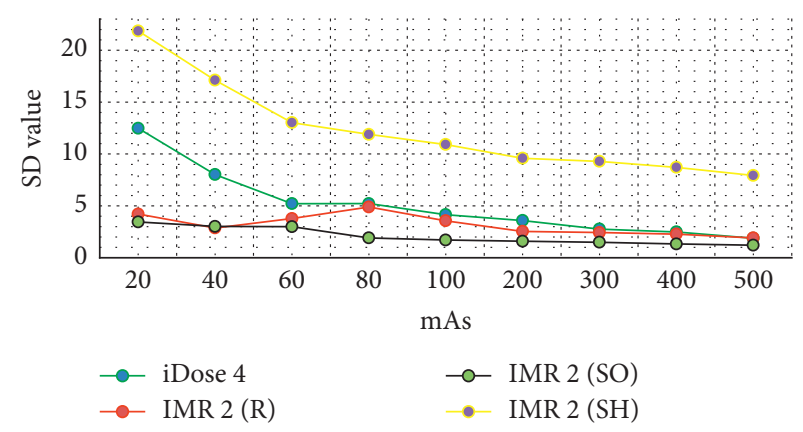

FIgURE 5: Comparison of image SD values of the two algorithms. (R) denotes Routine, (SO) denotes Soft, and (SH) denotes SharpPlus.

diagnosis of benign and malignant pulmonary nodules before CT image processing were 59.4\%, 68.1\%, and 0.632, respectively. The diagnostic sensitivity, specificity, and AUC of CT images reconstructed by iDose 4 algorithm for benign and malignant lung nodules were $82.1 \%, 75.3 \%$, and 0.695 , respectively. Those of CT images reconstructed by IMR2 algorithm for benign and malignant pulmonary nodules 
were $85.7 \%, 82.3 \%$, and 0.815 , respectively, which were significantly higher than the original CT images $(P<0.05)$.

\subsection{Comparison of CT Imaging Information of Two Groups of} Patients Processed by IMR 2 Algorithm. The CT images and other information of 96 patients with lung cancer are summarized in Figures 8-10. Figure 8 shows the distribution of lung cancer types, Figure 9 shows the comparison of tumor size between the two groups, and Figure 10 shows the comparison of CT imaging features between the two groups. There were 65 cases of malignant nodules in lung cancer patients, including 15 cases of squamous cell carcinoma, 31 cases of adenocarcinoma, and 19 cases of small cell carcinoma. Of which, 31 cases were benign nodules, including 14 hamartomas, 10 tuberculous granulomas, 2 sclerosing hemangiomas, and 5 diffuse lymphocyte hyperplasia. The average size of lung nodules in the malignant group and benign group of lung cancer patients was $23(15,27) \mathrm{mm}$ and $14(11,19) \mathrm{mm}$, respectively. The size of benign nodules was significantly smaller than that of malignant nodules $(P=0.012)$. In the malignant group of lung nodules, the morphology of the nodules was irregular. The proportions of burr sign, lobular sign, vascular sign, bronchial sign, and pleural depression sign were $92 \%, 48 \%, 42 \%, 45 \%, 38 \%$, and $43 \%$, respectively. In addition, the percentages of indications in the benign pulmonary nodule group were $59 \%, 29 \%, 19 \%$, $27 \%, 18 \%$, and $20 \%$, respectively. There were significant differences in the number of patients with each indication between the two groups $(P<0.05)$ (Figure 10).

\section{Discussion}

Chest CT lung cancer screening is the best way to detect early lung cancer [11]. At present, the use of chest CT for preoperative diagnosis of lung cancer is relatively common, but due to the large image noise and other shortcomings, the traditional method of using the subjective judgment of physicians is likely to be misdiagnosed $[12,13]$. Therefore, how to accurately locate the tumor lesions still needs further research. The advent of iterative reconstruction algorithms has greatly reduced image noise. With the advancement of computer software and hardware technology, it has been widely used clinically. In the evaluation system of CT image quality, image noise (SD, SNR, and CNR) is only one aspect, and its core evaluation indicators are the spatial resolution and density resolution of the image. Studies found that the image quality of images reconstructed by iDose reconstruction and conventional dose scan FBP reconstruction has no difference in image quality and lower noise [14]. In addition, there were reports that the IMR algorithm [15], as an emerging full model iterative reconstruction technology, was mainly used in computer-aided medical imaging diagnosis and analysis to evaluate the efficacy of radiotherapy, image-based pathological analysis, tumor microenvironment, and heterogeneity research. For example, the highthroughput processing of multimodal images and the analysis of graphics into data can provide quantification of diagnosis basis and have powerful image noise reduction

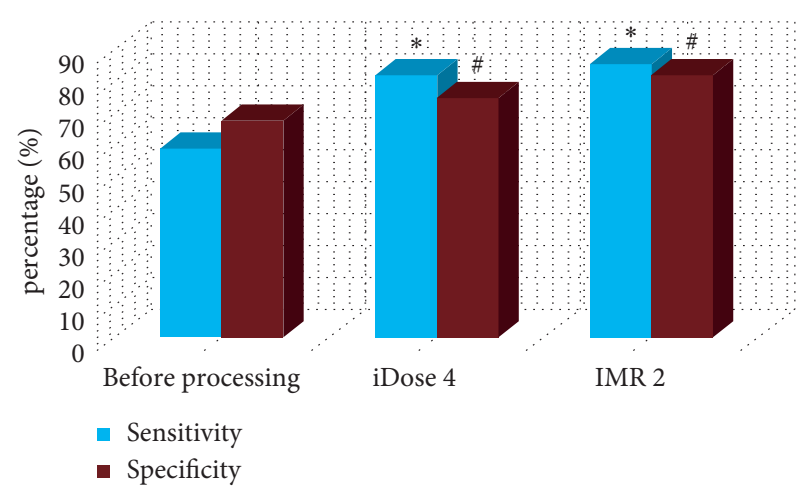

FIgURE 6: Comparison of sensitivity and specificity of different algorithms for lung cancer detection. * indicates that the sensitivity was considerably different versus that before treatment $(P<0.05)$; \# indicates that the specificity was remarkably different versus that before treatment $(P<0.05)$.

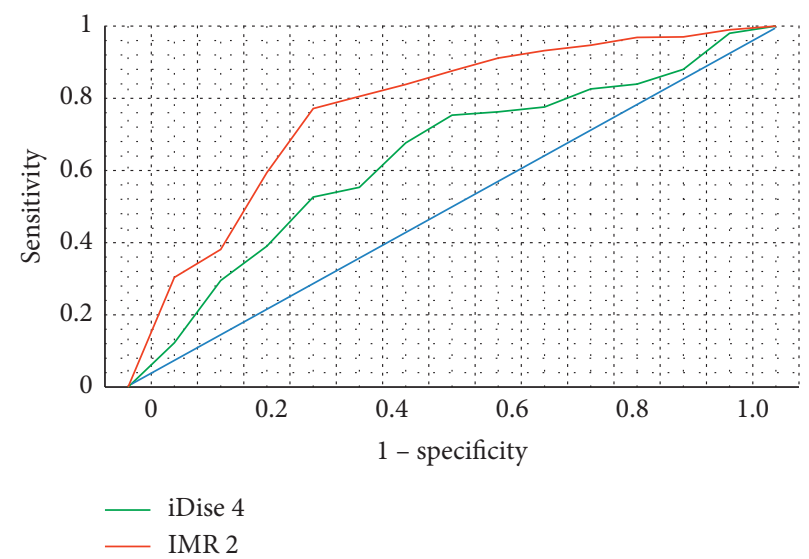

FIGURE 7: Comparison of AUC area of patient CT images processed by two algorithms.

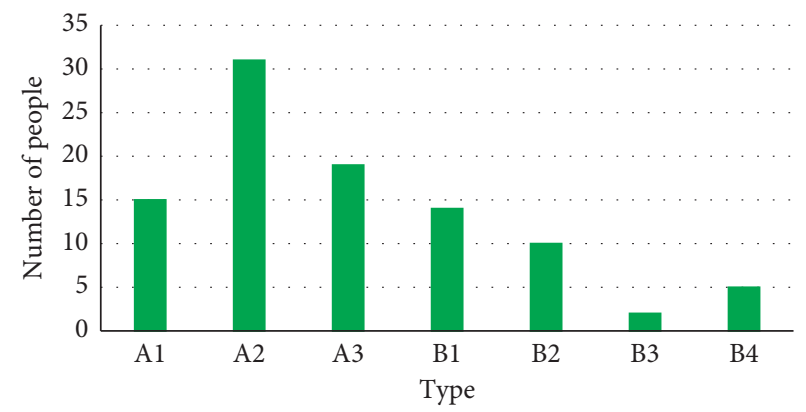

FIGURE 8: Distribution of lung cancer types in patients. A: malignant nodule, B: benign nodule, A1: squamous cell carcinoma, A2: adenocarcinoma, A3: small cell carcinoma, B1: hamartoma, B2: tuberculous granuloma, B3: sclerosing hemangioma, and B4: diffuse lymphocyte proliferation.

capabilities [16, 17]. Based on traditional chest CT, the image quality using the IMR algorithm is good and the noise is low. Therefore, LDCT scan $(120 \mathrm{kV}, 30 \mathrm{mAs})$ was adopted in this research, and the image reconstructed by iDose 4 was set as a 


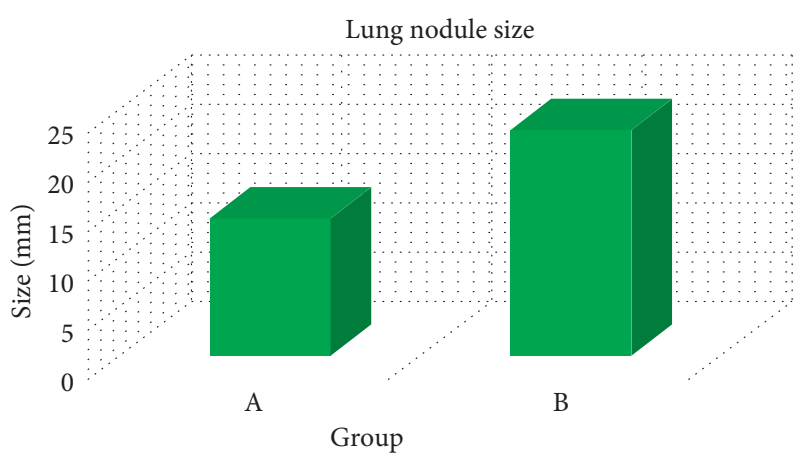

Figure 9: Comparison of tumor size between two groups of patients. A: benign nodule; $\mathrm{B}$ malignant nodule.

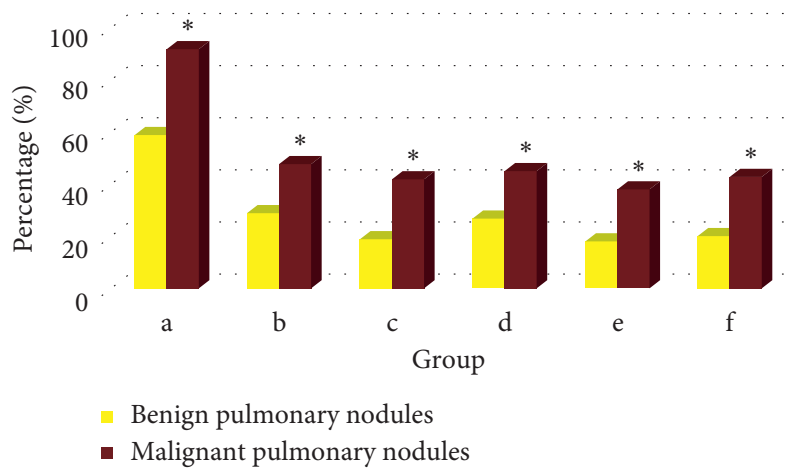

Figure 10: Comparison of CT imaging features between the two groups. a denotes irregular nodule morphology, b denotes burr sign, $\mathrm{c}$ denotes lobular sign, d denotes vascular sign, e denotes bronchial sign, and $\mathrm{f}$ meant denotes depression sign; * indicates that the difference was considerable versus that of benign group of patients $(P<0.05$.$) .$

reference to compare and study the ability of IMR iterative reconstruction algorithm to improve image quality. Moreover, the chest CT accuracy, spatial resolution, density resolution, image uniformity [18], and noise were evaluated.

The results showed that the spatial resolution, density resolution, image uniformity, and contrast of the CT image reconstructed by the IMR2 algorithm were significantly greater than that of the iDose4 algorithm, and the noise was significantly less than that of the iDose4 algorithm $(P<0.05)$. The subject ROC curve was used to analyze the diagnostic sensitivity, specificity, and AUC of benign and malignant pulmonary nodules based on CT. It was found that the diagnostic sensitivity, specificity, and AUC of CT images reconstructed by IMR2 algorithm for benign and malignant lung nodules were significantly higher than that of original CT images $(P<0.05)$. In short, chest $\mathrm{CT}$ based on the IMR2 algorithm can significantly improve the diagnosis of lung cancer and was of practical significance for the timely detection of early lung cancer. This was consistent with the findings of Lombard et al. [19]. In addition, the patients were grouped according to the benign and malignant conditions of different lung nodules, and the differences in chest CT imaging characteristics between the two groups were compared. It was found that the malignant pulmonary nodule group and the benign pulmonary nodule group had statistical differences in lung nodule size, nodule morphology, burr sign, lobular sign, vascular sign, bronchial sign, and pleural depression sign $(P<0.05)$. It was suggested that the benign and malignant conditions of lung nodules in patients with lung cancer were closely related to the imaging features presented by CT [20]. The tumor size in chest CT images of patients with malignant pulmonary nodules was significantly larger than that in the benign nodules group. The proportion of patients with irregular nodules, burr sign, lobular sign, vascular sign, bronchial sign, and pleural sign was significantly higher than that of the benign group. In summary, chest CT based on the full model iterative reconstruction algorithm had a good diagnostic value for lung cancer patients, can significantly improve the diagnostic efficiency of lung cancer, and was worthy of clinical promotion.

\section{Conclusion}

To explore the application of IMR processed chest CT images in the clinical diagnosis of lung cancer patients, the iDose4 algorithm was employed and applied to the CT image analysis of 96 lung cancer patients. It was found that the IMR algorithm showed high accuracy and AUC area for lung cancer diagnosis and prediction compared with the iDose4 algorithm for processing patient CT images. Among them, IMR2 algorithm combined with SharpPlus parameters can be used as the optimal feature combination model for the diagnosis of lung cancer. However, the selection of patient samples is small and the source is single in this study, and the different manifestations of lung cancer patients are not discussed in detail. It is impossible to verify the impact of these features on the accuracy of the prediction. In the future, it will consider increasing the sample size of lung cancer patients and further adopting a multicenter collaborative analysis method for research. In conclusion, the results provide good clinical data support and certain theoretical support for the application of the IMR algorithm in the clinical diagnosis of lung cancer.

\section{Data Availability}

The data used to support the findings of this study are available from the corresponding author upon request.

\section{Conflicts of Interest}

The authors declare no conflicts of interest.

\section{Authors' Contributions}

Jie Li and Wei Wang contributed equally to this work.

\section{Acknowledgments}

This work was supported by Zhuhai Science and Technology Plan Medical Project (20181117A010054). 


\section{References}

[1] D. Groheux, G. Quere, E. Blanc et al., "FDG PET-CT for solitary pulmonary nodule and lung cancer: literature review," Diagnostic and Interventional Imaging, vol. 97, no. 10, pp. 1003-1017, 2016, Epub 2016 Aug 24. PMID: 27567555.

[2] B. C. Bade and C. S. Dela Cruz, "Lung cancer 2020," Clinics in Chest Medicine, vol. 41, no. 1, pp. 1-24, 2020, PMID: 32008623.

[3] A. Kandathil, F. U. Kay, Y. M. Butt, J. W. Wachsmann, and R. M. Subramaniam, "Role of FDG PET/CT in the eighth edition of TNM staging of non-small cell lung cancer," $R a$ dioGraphics, vol. 38, no. 7, pp. 2134-2149, 2018, PMID: 30422775.

[4] J. H. Pedersen, Z. Saghir, M. M. Wille, L. H. Thomsen, B. G. Skov, and H. Ashraf, "Ground-glass opacity lung nodules in the era of lung cancer CT screening: radiology, pathology, and clinical management," Oncology, vol. 30, no. 3, pp. 266-274, 2016, PMID: 26984222.

[5] R. C. Delgado Bolton, A. K. Calapaquí-Terán, F. Giammarile, and D. Rubello, "Role of 18F-FDG-PET/CT in establishing new clinical and therapeutic modalities in lung cancer. a short review," Revista Española de Medicina Nuclear e Imagen Molecular, vol. 38, no. 4, pp. 229-233, 2019, English, Spanish Epub 2019 Jun 13. PMID: 31202725.

[6] G. Laurent, N. Villani, G. Hossu et al., "Full model-based iterative reconstruction (MBIR) in abdominal CT increases objective image quality, but decreases subjective acceptance," European Radiology, vol. 29, no. 8, pp. 4016-4025, 2019, Epub 2019 Jan 30. PMID: 30701327.

[7] T. Hamamura, Y. Hayashida, Y. Takeshita et al., "The usefulness of full-iterative reconstruction algorithm for the visualization of cystic artery on CT angiography," Japanese Journal of Radiology, vol. 37, no. 7, pp. 526-533, 2019.

[8] D. Zeng, L. Yao, Y. Ge et al., "Full-spectrum-knowledge-aware tensor model for energy-resolved ct iterative reconstruction," IEEE Transactions on Medical Imaging, vol. 39, no. 9, pp. 2831-2843, 2020, Epub 2020 Feb 27. PMID: 32112677.

[9] J. Sun, Q. Zhang, X. Duan et al., "Application of a full modelbased iterative reconstruction (MBIR) in $80 \mathrm{kVp}$ ultra-lowdose paranasal sinus CT imaging of pediatric patients," $\mathrm{La}$ radiologia medica, vol. 123, no. 2, pp. 117-124, 2018, Epub 2017 Oct 10. PMID: 29019028.

[10] J. Greffier, J. Frandon, A. Larbi, J. P. Beregi, and F. Pereira, "CT iterative reconstruction algorithms: a task-based image quality assessment," European Radiology, vol. 30, no. 1, pp. 487-500, 2020, Epub 2019 Jul 29. PMID: 31359122.

[11] A. Kandathil, R. C. S. Iii III, and R. M. Subramaniam, "Lung cancer recurrence: 18F-FDG PET/CT in clinical practice," American Journal of Roentgenology, vol. 213, no. 5, pp. 1136-1144, 2019, Epub 2019 Jul 30. PMID: 31361525.

[12] T. Nawa, "Low-dose CT screening for lung cancer reduced lung cancer mortality in Hitachi City," International Journal of Radiation Biology, vol. 95, no. 10, pp. 1441-1446, 2019, Epub 2018 Oct 11. PMID: 30307383.

[13] R. H. Wang, K. Xu, L. Li, and Z. F. Wu, "Lung cancer combined with connective tissue disease-related interstitial lung disease: CT features," Zhonghua Zhongliu Zazhi, vol. 42, no. 8, pp. 665-669, 2020, Chinese PMID: 32867459.

[14] M. E. Clark, L. E. Bedford, B. Young et al., "Lung cancer CT screening: psychological responses in the presence and absence of pulmonary nodules," Lung Cancer, vol. 124, pp. 160-167, 2018, Epub 2018 Aug 3. PMID: 30268456.
[15] O. Leleu, D. Basille, M. Auquier et al., "Lung cancer screening by low-dose CT scan: baseline results of a French prospective study," Clinical Lung Cancer, vol. 21, no. 2, pp. 145-152, 2020, Epub 2019 Oct 31. PMID: 31982356.

[16] G. Shirota, E. Maeda, Y. Namiki et al., "Pediatric 320-row cardiac computed tomography using electrocardiogramgated model-based full iterative reconstruction," Pediatric Radiology, vol. 47, no. 11, pp. 1463-1470, 2017, Epub 2017 Jun 30. PMID: 28667349; PMCID: PMC5608791.

[17] L. Hehn, R. Gradl, M. Dierolf, K. S. Morgan, D. M. Paganin, and F. Pfeiffer, "Model-based iterative reconstruction for propagation-based phase-contrast X-ray CT including models for the source and the detector," IEEE Transactions on Medical Imaging, vol. 39, no. 6, pp. 1975-1987, 2020, Epub 2019 Dec 26. PMID: 31880549.

[18] Y. Funama, D. Utsunomiya, K. Hirata et al., "Improved estimation of coronary plaque and luminal attenuation using a vendor-specific model-based iterative reconstruction algorithm in contrast-enhanced CT coronary angiography," Academic Radiology, vol. 24, no. 9, pp. 1070-1078, 2017, Epub 2017 Apr 7. PMID: 28396126; PMCID: PMC5557674.

[19] C. Lombard, A. Gervaise, N. Villani et al., "The impact of dose reduction in quantitative kinematic $\mathrm{CT}$ of ankle joints using a full model-based iterative reconstruction algorithm: a cadaveric study," American Journal of Roentgenology, vol. 210, no. 2, pp. 396-403, 2018, Epub 2017 Dec 12. PMID: 29231760.

[20] K. Yasaka, T. Furuta, T. Kubo et al., "Full and hybrid iterative reconstruction to reduce artifacts in abdominal CT for patients scanned without arm elevation," Acta Radiologica, vol. 58, no. 9, pp. 1085-1093, 2017, Epub 2017 Jan 9. PMID: 28068822 . 